\title{
Siglec-7 on peripheral blood eosinophils: surface expression and function
}

\author{
Fanny Legrand, PhD, PharmD ${ }^{\mathrm{a}, 1}$, Nadine Landolina, PharmD, PhD ${ }^{\mathrm{b}, 1}$, Ilan Zaffran, MSc ${ }^{\mathrm{b}}$, \\ Robert O. Emeh, BS ${ }^{a}$, Elizabeth Chen ${ }^{a, c}$, Amy D. Klion, MD ${ }^{a, 2}$, Francesca Levi-Schaffer, \\ PharmD, PhD ${ }^{\mathrm{b}, 2}$ \\ aHuman Eosinophil Section, Laboratory of Parasitic Diseases, National Institute of Allergy and \\ Infectious Diseases, MD, USA; \\ bPharmacology \& Experimental Therapeutics Unit, Institute for Drug Research, School of \\ Pharmacy, Faculty of Medicine, The Hebrew University of Jerusalem, Israel; \\ ${ }^{\circ}$ Current affiliation: University of Maryland, USA
}

\begin{abstract}
Background: Siglec-7 is an inhibitory receptor (IR) expressed on human blood eosinophils. Whereas activation of other IRs, including Siglec-8 and CD300a, has been shown to downregulate eosinophil function, little is known about the role of Siglec-7 on human eosinophils.

Objective.-To examine Siglec-7 expression and function in eosinophils from normal (ND) and eosinophilic (EO) donors.

Methods: Eosinophil expression of Siglec-7 was quantified by flow cytometry and quantitative PCR. Soluble Siglec-7 (sSiglec-7) levels were measured by ELISA in serum. The effect of Siglec-7 on eosinophil viability and degranulation was assessed in vitro by AnnexinV-FITC/7AAD staining and by measuring GM-CSF-induced mediator release in culture supernatants. Signal transduction was studied by Western Blot.

Results: Siglec-7 was expressed ex vivo on blood eosinophils from all eosinophilic and normal individuals studied. Siglec-7 surface, but not SIGLEC-7 mRNA expression, was correlated with absolute eosinophil count (AEC). Siglec-7 was upregulated on purified eosinophils after in vitro stimulation with GM-CSF or IL-5. Serum sSiglec-7 was detectable in 133/144 subjects tested and correlated with AEC. Siglec-7 crosslinking inhibited GM-CSF-induced release of eosinophil peroxidase, TNFa and IL-8 ( $n=7-8)$ but did not promote eosinophil apoptosis $(n=5)$. Finally,
\end{abstract}

\footnotetext{
Corresponding Author: Fanny Legrand, PhD, PharmD, Human Eosinophil Section, Laboratory of Parasitic Diseases, National Institute of Allergy and Infectious Diseases, MD, USA, Fanny.legrand@nih.gov.

$1_{\text {These authors contributed equally. }}$

2 These authors jointly supervised this work.

Author contributions

F.L., N.L., I.Z., R.O.E. and E.C. performed all the laboratory experiments and F.L., N.L. analyzed the data and drafted the manuscript. A.K. and F.L.S. designed the study revised and corrected the manuscript, obtained funding for the study. All authors reviewed and approved the final version of the manuscript.

Competing interests

None of the authors report any conflicts of interest related to this work.
} 
Siglec-7 crosslinking on GM-CSF-activated eosinophils induced phosphorylation of SHP-1 and de-phosphorylation of ERK $1 \frac{1}{2}$ and $\mathrm{p} 38$.

Conclusions: Siglec-7 is constitutively expressed on human eosinophils and downmodulates eosinophil activation. Targeting of Siglec-7 on eosinophils might enhance treatment efficacy in eosinophil-driven disorders. Conversely, therapeutic interventions that inhibit Siglec-7 could have unanticipated consequences and promote eosinophilic inflammation.

\section{Capsule Summary}

Siglec-7 is an inhibitory receptor expressed on human blood eosinophils. Siglec-7 activation downregulates eosinophil activation but does not affect survival. Targeting of Siglec-7 on eosinophils could be useful in the treatment of eosinophilic inflammation.

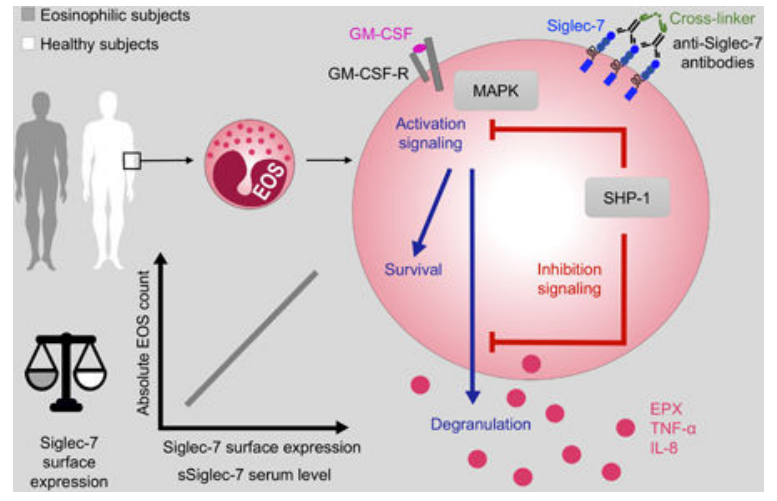

\section{Keywords}

apoptosis; eosinophilia; eosinophils; hypereosinophilic syndrome; ITIM; Siglec-7; Siglec-8; signaling

\section{Introduction}

Eosinophils are main effector cells in allergic inflammation and have been implicated in the late and chronic phases of the allergic response. Their recruitment and activation are regulated by a complex network of activating and inhibitory signals that represent key therapeutic targets ${ }^{1,2}$. Initial attention focused primarily on the IL-5 axis in eosinophilassociated disorders, leading to the recent approval of mepolizumab, reslizumab and benralizumab for the treatment of eosinophilic asthma ${ }^{3,4}$ and mepolizumab for the treatment of eosinophilic granulomatosis with polyangiitis ${ }^{5}$. The role of inhibitory receptors (IRs), many of which are shared by eosinophils, basophils and mast cells, has been less wellexplored.

IRs, including members of the Sialic acid binding Ig-like lectin (Siglec) family ${ }^{6}$ and the Iglike superfamily member $\mathrm{CD} 300 \mathrm{a}^{7}$, are characterized by immunoreceptor tyrosine-based inhibitory motifs (ITIMs) in their cytoplasmic tail, which, when phosphorylated, decrease cellular activation and/or induce apoptosis. The precise effects of an individual IR may vary depending on the cell type. For example, crosslinking of Siglec-8, an IR expressed on eosinophils ${ }^{8}$, mast cells ${ }^{9}$ and basophils ${ }^{10}$, leads to apoptosis of eosinophils ${ }^{11,12}$, but inhibits 
degranulation of mast cells without promoting cell death ${ }^{9}$. In contrast, CD300a activation causes decreased function and apoptosis of both mast cells and eosinophils in the presence of their respective growth factors ${ }^{13,14}$.

Originally detected on Natural Killer cells (NK cells) and monocytes ${ }^{15,16}$, Siglec-7 (p75/ AIRM) is an additional inhibitory member of the Siglec family that is expressed on a wide variety of cells, including human eosinophils ${ }^{13}$, mast cells and basophils ${ }^{17}$. Siglec-7 displays two signaling motifs: a membrane-proximal ITIM and a membrane-distal motif (ITIMlike) ${ }^{15}$. On NK cells, the Siglec-7 cytoplasmic domain associates with the protein tyrosine phosphatases Src homology-2 (SH2) domain-containing protein-tyrosine phosphatase-1 (SHP-1), which mediates the Siglec-dependent inhibitory signal ${ }^{18}$.

In contrast to Siglec- 8 which binds sialylated ligands on human airways cells ${ }^{19}$, Siglec-7 binds preferentially to a 2,8-disialyl and branched a2,6-sialyl carbohydrate structures ${ }^{20}$, which are expressed on various tumor cells and normal colonic epithelial cells ${ }^{21}$. The function of Siglec-7 has been best studied in the context of tumor biology, where expression of Siglec-7 ligands on tumor cells suppress NK cell function leading to increased metastatic potential $^{22}$. These findings have stimulated the development of novel strategies to inhibit Siglec-7 function, including the development of low molecular weight, high affinity Siglec-7 ligands that block the inhibitory effects of tumor cells on NK cells in vitro ${ }^{23}$. Such strategies may have unanticipated consequences on cells other than NK cells that express Siglec-7, including the cells involved in allergic inflammation. Although activation of Siglec-7 on mast cells can inhibit IgE-mediated mast cell and basophil activation ${ }^{17}$, the function of Siglec-7 on eosinophils is unknown. The aim of the present study was to characterize the expression of Siglec-7 on eosinophils from normal donors (ND) and subjects with eosinophilia (EO) and to explore the functional consequences of Siglec-7 engagement on eosinophils.

\section{Materials and Methods}

\section{Study subjects}

Eosinophilic subjects (EO) underwent detailed clinical evaluation at the NIH Clinical Center as part of an NIAID Institutional Review Board (IRB)-approved protocol to study eosinophilic disorders (NCT00001406). For the purposes of this study, hypereosinophilic syndrome (HES) is defined as hypereosinophilia (AEC $\geq 1500 / \mu \mathrm{L})$ with evidence of eosinophil-associated clinical manifestations. EO included subjects with idiopathic HES $(n=32)$, myeloid HES ( $n=19)$, lymphocytic HES $(n=15)$, overlap HES $(n=30)$, hypereosinophilia of unknown significance $(n=8)$, familial eosinophilia $(n=3)$, episodic angioedema and eosinophilia $(n=3)$, and hypereosinophilia associated with neoplasia $(n=5)$, immunodeficiency $(n=3)$ and atopy $(n=1)$. Forty-seven subjects were untreated at the time of analysis (geometric mean (GM) AEC 2505/ $\mu \mathrm{L}$, range 470-68220/ $\mu \mathrm{L}$ ) and 72 were receiving treatment at the time of the study (GM AEC $871 / \mu \mathrm{L}$, range 30-26420/ $\mu \mathrm{L}$ ). See Supplementary Table 1 for details. Normal donors (ND) were recruited under NIAID IRB (NCT00090662) and Hadassah-Hebrew University Human Experimentation Helsinki Committee approved protocols for in vitro research. All participants gave written informed consent. 


\section{Eosinophil purification}

Eosinophils were purified by sedimentation on Ficoll-Hypaque and magnetic bead purification, as previously described ${ }^{13,24}$. Eosinophil purity was $>98 \%$ in all experiments with a viability of $>98 \%$, as assessed by trypan blue staining.

\section{Real-time quantitative PCR}

Total RNA was extracted from $10^{7}$ purified eosinophils using TriZol (Invitrogen) prior to cDNA synthesis using High Capacity cDNA Reverse Transcription Kit (Applied Biosystems, Carlsbad, Calif), according to the manufacturer's protocol. SIGLEC-7 and SIGLEC-8 mRNA, and 18S rRNA were amplified in a 96-well plate using the following commercially available TaqMan primers (Applied Biosystems) in a final volume of $10 \mu \mathrm{L}$ : Hs01100854_m1, Hs00274289_m1, and X03205.1, respectively. Each sample was run in triplicate, and cycle threshold levels were normalized using the $18 \mathrm{~S}$ cycle threshold values from corresponding samples. mRNA levels are expressed in arbitrary units $\left(\mathrm{x}_{10}^{-5}\right)$ related to $18 \mathrm{~S}$ rRNA.

\section{Assessment of surface receptor expression by flow cytometry on whole blood}

Surface expression of Siglec-7 was quantified by multiparameter flow cytometry ex vivo on eosinophils (CD45 ${ }^{+} \mathrm{CD} 16^{-}$granulocytes) in whole blood as previously described ${ }^{24}$ and in vitro using purified blood eosinophils $\left(2 \times 10^{6} / \mathrm{mL}\right)$ incubated with or without $10 \mathrm{ng} / \mathrm{mL}$ of rhIL-5 (R\&D, Minneapolis, MN, USA) or rhGM-CSF (PeproTech, Rocky Hill, NJ, USA). The antibodies used are provided in the online Supplementary Materials and Methods.

\section{Modulation of Siglec-7 expression on purified eosinophils in vitro}

Purified eosinophils $\left(2 \times 10^{6} / \mathrm{mL} /\right.$ wells) were incubated overnight with or without $10 \mathrm{ng} / \mathrm{mL}$ of recombinant human cytokines (rhGM-CSF, rhIL-5, rhIL-33, rhIFN- $\alpha$, rhIFN- $\gamma$ ), SEB (5 $\mu \mathrm{g} / \mathrm{mL}$ ) or $E$. coli extracts $(4 \mu \mathrm{g} / \mathrm{mL})$. Surface expression of Siglec-7 was determined by flow cytometry on viable eosinophils (7-AAD ${ }^{\text {neg }}$ ), and RNA was extracted from TriZol and mRNA levels assessed after overnight incubation with and without GM-CSF (10 ng/mL).

\section{Measurement of soluble Siglec-7 receptor levels in serum.}

Serum levels of soluble Siglec-7 (sSiglec-7) were quantified using a commercially available sandwich ELISA (Human Siglec-7/CD328 DuoSet ELISA, R\&D) according to the manufacturer's instructions. All assays were performed in singlicate using undiluted serum, and values were calculated on the basis of a recombinant standard curve. The minimal level of detection of sSiglec-7 in serum was $125 \mathrm{pg} / \mathrm{mL}$.

\section{In vitro eosinophil stimulation and inhibition assays}

Freshly isolated eosinophils $\left(1.5 \times 10^{5} / 150 \mu \mathrm{L}\right.$ in culture medium $)$ were blocked in $5 \%$ goat serum in PBS prior to incubation for 30 minutes at $4^{\circ} \mathrm{C}$ in 96 -well $\mathrm{U}$ shape plates (Nunc, Roskilde, Denmark) in the presence of anti-Siglec-7 (QA79, eBiosciences), anti-Siglec-8 (7C9, Biolegend), or matched control antibodies (mouse $\mathrm{IgG1k}$ isotype control, eBiosciences) $(0.62-5 \mu \mathrm{g} / \mathrm{mL})$. After washing, crosslinker $\left(\mathrm{F}\left(\mathrm{ab}^{\prime}\right)_{2}\right.$ fragment goat anti-mouse $\operatorname{IgG}(\mathrm{H}+\mathrm{L})(10 \mu \mathrm{g} / \mathrm{mL})$, Jackson Laboratories, West Grove, PA, US) and rhGM-CSF (50 
$\mathrm{ng} / \mathrm{mL}$ ) were added simultaneously and the cells were incubated for 40 minutes (eosinophil peroxidase, EPX release) or overnight (CD69 expression and cytokine release) at $37^{\circ} \mathrm{C}, 5 \%$ $\mathrm{CO}_{2}$ in phenol-free RPMI 5\% FCS. Supernatants were collected and stored at $-20^{\circ} \mathrm{C}$. AntiCD300a ( $1 \mu \mathrm{g} / \mathrm{mL}$, from hybridoma \#12, provided by Prof. O. Mandelboim) was used as a positive control for inhibition in all experiments.

\section{Measurement of EPX and cytokines in supernatants}

EPX release was measured by colorimetric assay using freshly prepared peroxidase substrate solution containing o-phenylenediamine (OPD) as described previously ${ }^{25}$. A standard curve was constructed using purified human EPX (provided by Dr. Gerald J. Gleich, University of Utah Health Sciences Center, Salt Lake City, UT, USA). The reaction was stopped by the addition of $100 \mu \mathrm{l}$ of $4 \mathrm{mM}$ sulfuric acid (BDH), and absorbance was determined at $492 \mathrm{~nm}$ in a spectrophotometer (PowerWave XS; Bio-Tek Instruments). IL-8 and TNFa levels were detected in supernatants by ELISA (Peprotech, minimum detectable level $16 \mathrm{pg} / \mathrm{mL}$ ). Data are expressed as \% of control, calculated by dividing the concentration in the antibodytreated well by that in the corresponding uninhibited control well (GM-CSF only) and multiplying by 100 .

\section{Assessment of eosinophil apoptosis.}

Eosinophil cell death was quantified by flow cytometry using AnnexinV-FITC/7-AAD staining (BD, San Jose, CA, USA). Briefly, after overnight incubation with the cytokines (GM-CSF or IL-5 at $10 \mathrm{ng} / \mathrm{mL}$ ), anti-Siglec-7, anti-Siglec-8 or their respective isotype controls were added for another overnight incubation at $37^{\circ} \mathrm{C}, 5 \% \mathrm{CO}_{2}$. The cells were then washed in PBS $1 \mathrm{X}$, stained with AnnexinV-FITC and 7-AAD probes ( $5 \mu \mathrm{L} /$ tube $)$ in a binding buffer (BD) during 15 minutes room temperature. The samples were acquired on a LSRII flow cytometer (BD).

\section{Western Blot}

Crosslinking with anti-Siglec-7, anti-CD300a or matched control antibody was performed on freshly isolated peripheral blood eosinophils $\left(2 \times 10^{6} / 200 \mu \mathrm{L}\right)$ in culture medium for 30 minutes at $4^{\circ} \mathrm{C}$ as described above. Cells were activated for the indicated times in Eppendorf tubes in a $37^{\circ} \mathrm{C}$ water bath in the presence of rhGM-CSF $(50 \mathrm{ng} / \mathrm{mL})$ and sheep anti-mouse $\mathrm{F}\left(\mathrm{ab}^{\prime}\right) 2$ crosslinker antibodies $(\mathrm{CL}),(10 \mu \mathrm{g} / \mathrm{mL})$. Activation was stopped by adding ice-cold PBS to the pellet followed by centrifugation. Cell pellets were lysed using a cell lysis buffer (\#7018 Cell Signaling Technology, Danvers, MA) supplemented with protease inhibitor cocktail (1:100, Sigma-Aldrich, Rehovot, Israel), and cell debris was removed from the lysates by centrifugation $\left(14000 \mathrm{~g}, 1\right.$ minute $\left.4^{\circ} \mathrm{C}\right)$. Western blot analysis was performed using standard techniques (see Supplement for details).

\section{Statistical analysis}

Group means were compared using the Mann-Whitney U test, ANOVA (for multiple group comparisons) and Wilcoxon signed rank test (for paired analyses). Spearman rank was used for determination of correlation and a custom rank trend test for Western blot analysis (see Supplemental Methods). $P$ values less than .05 were considered significant for all analyses. 


\section{Results}

\section{Eosinophil expression of Siglec-7}

Siglec-7 expression was detected on eosinophils from all 23 ND and 31 EO subjects tested. Geometric mean (GM) Siglec-7 expression showed a tendency towards an increase in EO subjects compared to ND with GM $\triangle \mathrm{MFI}$ of 183 vs 105 , respectively, $(\mathrm{P}=0.065$; Figure 1A) and was significantly correlated with absolute eosinophil count (AEC) $(\mathrm{n}=52, \mathrm{P}<0.05$, Figure 1B). Siglec-7 was expressed on blood eosinophils at lower levels than the inhibitory receptors, CD300a and Siglec-8 (GM $\triangle$ MFI 271 vs 667 and 980, respectively, n=20 EO; GM $\triangle$ MFI 307 vs 852 vs 837, n=11 ND; Wilcoxon test, $\mathrm{P}<0.05$ to $\mathrm{P}<0.0001$; Figure $1 \mathrm{C}$ ) and was positively correlated with Siglec- 8 expression $(r=+0.52, \mathrm{P}<0.0001, \mathrm{n}=47$; Figure 1D) and CD300a ( $r=+0.7, P<0.0001, n=27$, Supplementary Figure 1).

SIGLEC-7mRNA was quantified in purified peripheral blood eosinophils from 45 subjects (13 ND and $32 \mathrm{EO}$ ) and levels were detectable in all subjects tested (data not shown). SIGLEC-7 mRNA levels were not correlated with the surface Siglec-7 levels measured on blood eosinophils by flow cytometry (Figure 1E). Moreover, contrary to what was seen at the cell surface levels by flow cytometry (Figure $1 \mathrm{D}$ ), levels of mRNA for SIGLEC-7 and SIGLEC-8 were not correlated (Figure 1F).

\section{Measurement of soluble Siglec-7 receptor levels in serum}

sSiglec-7 was measured by ELISA in the serum of normal and eosinophilic donors ( $\mathrm{n}=16$ and 126) and was detectable in 133 of 142 subjects tested. Compared to ND (GM 270 $\mathrm{pg} / \mathrm{mL} ; \mathrm{n}=16$ ) and treated EOS patients (GM $366 \mathrm{pg} / \mathrm{mL} ; \mathrm{n}=81$ ), serum sSiglec-7 levels were increased in untreated EOS subjects (GM $501 \mathrm{pg} / \mathrm{mL} ; \mathrm{n}=45$ ) (Figure 2A). Untreated EO subjects also had higher GM AEC than ND or treated patients (GM 2281 vs 250 vs 907 Eos/ $\mu \mathrm{L}$, Figure 2B). Serum levels of sSiglec-7 were weakly but significantly correlated with AEC (n=135, $r=+0.23, P<0.001$, Figure $2 \mathrm{C}$ ). However, there was no significant correlation between sSiglec-7 levels in serum and surface expression of Siglec-7 on blood eosinophils for the 32 subjects tested including $11 \mathrm{ND}$ and 21EOS ( $\mathrm{r}=-0.14, P=0.42$; Figure 2D). Levels of serum sSiglec-7 were comparable in untreated subjects of different HES subtypes, with the exception of MHES subjects who had significantly higher sSiglec-7 levels than ND (942 vs $270 \mathrm{pg} / \mathrm{mL}$ respectively, Figure 2E). Of note, GM AEC was the highest in this HES subgroup (Figure 2F), and sSiglec-7 levels and AEC were correlated $(\mathrm{r}=+0.6, P<0.05, \mathrm{n}=15$, data not shown).

\section{Modulation of Siglec-7 expression in vitro}

Geometric mean surface expression of Siglec-7 on purified blood eosinophils increased from an $\triangle$ MFI of 67.3 to 101.1 and 100.8 after an overnight incubation with GM-CSF or IL-5 (10 $\mathrm{ng} / \mathrm{mL}$ ), respectively $(P<0.05, \mathrm{n}=7$; Figure 3A). Other stimuli, including IL-33, SEB (Figure $3 \mathrm{~A})$, E. Coli extracts, rhIFN-a and rhIFN- $\gamma$, had no detectable effect on Siglec-7 surface expression ( $\mathrm{n}=6$, Supplementary data Figure 2). Similarly, SIGLEC-7 mRNA levels significantly increased from 7.0 to 14.5 A.U. $\times 10^{-5}$ in purified eosinophils incubated overnight with GM-CSF $(P<0.05 ; \mathrm{n}=7$; Figure 3B $)$. 


\section{Effect of Siglec-7 engagement on eosinophil viability and function}

In contrast to anti-Siglec-8 antibodies, which induced eosinophil apoptosis, as assessed by total Annexin-V staining, after overnight priming with GM-CSF or IL-5 $(10 \mathrm{ng} / \mathrm{mL})$, antiSiglec-7 antibodies had no effect on eosinophil apoptosis under the same conditions $(n=5$; Figure 4 ). The $\%$ of $7-\mathrm{AAD}^{+}$eosinophils was also unchanged in response to anti-Siglec-7 (data not shown).

Crosslinking of Siglec-7 in the presence of GM-CSF reduced eosinophil activation in vitro, as assessed by surface expression of CD69 (Figure 5A, $\mathrm{n}=6, P<0.05$ ). Crosslinking of Siglec-7, but not Siglec-8, on eosinophils also significantly inhibited the release of EPX, TNFa, and IL-8 induced by GM-CSF ( $50 \mathrm{ng} / \mathrm{mL}$ ) compared to the isotype control (mIgG1) $(\mathrm{n}=7-8$; Wilcoxon test, Figure $5 \mathrm{~B}, \mathrm{C}, \mathrm{D})$.

\section{Activation of Siglec-7 on eosinophils induces SHP-1 phosphorylation and decreases MAPK phosphorylation in a time-dependent fashion}

Siglec-7 crosslinking of GM-CSF-activated eosinophils showed a trend towards induction of a time-dependent phosphorylation of SHP-1 (Figure 6A and 6B; $\mathrm{p}=0.054$, rank trend test). Conversely, GM-CSF induction of phosphorylation of ERK $1 \frac{1}{2}$ and $\mathrm{p} 38$ (Figure 6C, 6D and $6 \mathrm{E})$ were reduced by Siglec-7 crosslinking in a time-dependent manner, although this was not statistically significant. Notably, eosinophils incubated with anti-Siglec-7 alone displayed a basal phosphorylated state. Activation of CD300a (positive control) produced a pattern similar to that seen with Siglec-7.

\section{Discussion}

Inhibitory receptors (IRs) play important roles in modulating the function of innate immune cells, including eosinophils and mast cells, and have been proposed as therapeutic targets in allergic inflammation ${ }^{26}$. Siglec-7 is an IR expressed on a wide variety of human hematopoietic cells including NK cells, monocytes ${ }^{27}$, mast cells, basophils and eosinophils ${ }^{13,17}$. Decreased expression of Siglec-7 on selected cell populations has been reported in obesity ${ }^{28}$ and diabetes ${ }^{29}$ and has been associated with impaired NK cell function during chronic viral infections ${ }^{30,31}$. Although Siglec-7 expression was previously demonstrated on eosinophils ${ }^{13}$, the regulation of eosinophil expression of Siglec-7 in eosinophilic disorders and the functional consequences of Siglec-7 engagement on eosinophils has been largely unexplored.

In the present study, Siglec-7 expression was examined in normal donors and a large, heterogeneous cohort of eosinophilic subjects. Unlike Siglec-8, surface expression of Siglec-7 on eosinophils was positively correlated with absolute eosinophil count and was upregulated in vitro by IL-5 and GM-CSF, cytokines involved in eosinophilopoiesis and survival, but not by any of the other stimuli examined. These data, together with the finding that serum levels of sSiglec-7 are increased in eosinophilic donors, suggest that expression of Siglec-7 is actively regulated on the surface of eosinophils and may play a role in downmodulating eosinophil activation in the setting of allergic inflammation. 
In support of this hypothesis, antibody-induced Siglec-7 crosslinking inhibited GM-CSFmediated release of EPX, IL-8 and TNFa. This suppression of mediator release is similar to what has been described in response to crosslinking of Siglec-7 on anti-IgE-activated human cord blood derived mast cells ${ }^{17}$ and may be important in the context of the "allergic effector unit" where both mast cells and eosinophils interact via physical and soluble mechanisms ${ }^{32}$. A similar inhibition of mediator release has been reported with activation of the inhibitory receptor, CD300a, on eosinophils ${ }^{13}$ and may be explained by the ability of these two IRs to phosphorylate common pathways with a critical role in signal transduction, such as SHP-1 . In this context, the failure of Siglec-7 to induce eosinophil apoptosis (unlike CD300a) may be due to relatively weaker SHP-1 recruitment by Siglec-7 ${ }^{18}$. In contrast to Siglec-7 and CD300a, Siglec-8, which signals through a pathway involving Akt, p38, and c-Jun Nterminal kinase 1, appears to function predominantly as an activating receptor on IL-5 primed eosinophils leading to increased release of ROS and eosinophil apoptosis ${ }^{33}$.

The major limitation of the present study was the technical challenges of working with human eosinophils, which are present in relatively low numbers in peripheral blood of normal individuals and do not survive in culture without the addition of exogenous cytokines. Moreover, eosinophils purified from different individuals exhibit differences in the basal activation state. Unfortunately, Siglec-E, the murine ortholog of Siglec-7 and Siglec-9, is not expressed on murine eosinophils ${ }^{34}$ and humanized mouse models expressing Siglec-7 on eosinophils are unavailable at the present time.

In summary, Siglec-7 was constitutively expressed on eosinophils from all normal and eosinophilic donors tested, and expression was increased in the context of eosinophilia and by stimulation with cytokines that lead to eosinophilia and eosinophil activation.

Although the effect of Siglec-7 on eosinophil function was relatively modest, it appeared to be increased by eosinophil activation and was similar to the effect previously described on mast cell activation ${ }^{17}$. Whether targeting Siglec-7 (alone or in combination with other IRs) would be useful as an additional means of reducing allergic inflammation remains unknown. Conversely, the effects of agents currently in development designed to block Siglec-7 function in the setting of tumors may have unexpected effects on eosinophil and mast cell activation.

\section{Supplementary Material}

Refer to Web version on PubMed Central for supplementary material.

\section{Acknowledgments}

The authors would like to thank Michael Fay, Biostatistics Research Branch, NIAID, NIH for his assistance with the statistical analyses.

Funding

This study was funded in part by the Division of Intramural Research, NIAID, NIH. (AK) and in part by the Aimwell Charitable Trust Gutterman Funds and by the Israel Basic Science Foundation (FLS). 


\section{Abbreviations}

\begin{tabular}{|c|c|}
\hline AEC & Absolute eosinophil count \\
\hline CL & Crosslinker \\
\hline EO & Eosinophilic donors \\
\hline Eos & Eosinophils \\
\hline EPX & Eosinophil peroxidase \\
\hline E. Coli & Escherichia coli \\
\hline GM & Geometric mean \\
\hline GM-CSF & Granulocyte-macrophage colony-stimulating factor \\
\hline HES & Hypereosinophilic syndromes \\
\hline IL & Interleukin \\
\hline IR & Inhibitory receptor \\
\hline ITAM & Immunoreceptor tyrosine-based activating motifs \\
\hline ITIM & Immunoreceptor tyrosine-based inhibitory motif \\
\hline mAbs & Monoclonal Antibodies \\
\hline NK & Natural killer cells \\
\hline ND & Normal donors \\
\hline $\mathbf{R h}$ & Recombinant human \\
\hline RT & Room temperature \\
\hline SEB & Staphylococcus aureus Enterotoxin B \\
\hline SHP-1 & $\begin{array}{l}\text { Src homology-2 (SH2) domain-containing protein-tyrosine } \\
\text { phosphatase-1 }\end{array}$ \\
\hline Siglec & Sialic acid binding Ig-like lectin \\
\hline TNFa & Tumor necrosis factor alpha \\
\hline
\end{tabular}

\section{Bibliography}

1. Gangwar RS, Landolina N, Arpinati L, Levi-Schaffer F. Mast cell and eosinophil surface receptors as targets for anti-allergic therapy. Pharmacol Ther 2017;170:37-63. [PubMed: 27773785]

2. Legrand F, Klion AD. Biologic therapies targeting eosinophils: current status and future prospects. J Allergy Clin Immunol Pract 2015;3:167-74. [PubMed: 25754717]

3. Landolina N, Levi-Schaffer F. Monoclonal antibodies: the new magic bullets for allergy: IUPHAR Review 17. Br J Pharmacol 2016;173:793-803. [PubMed: 26620589] 
4. Farne HA, Wilson A, Powell C, Bax L, Milan SJ. Anti-IL5 therapies for asthma. Cochrane Database Syst Rev 2017;9:CD010834.

5. Wechsler ME, Akuthota P, Jayne D, Khoury P, Klion A, Langford CA, et al. Mepolizumab or Placebo for Eosinophilic Granulomatosis with Polyangiitis. N Engl J Med 2017;376:1921-32. [PubMed: 28514601]

6. Bochner BS, Zimmermann N. Role of siglecs and related glycan-binding proteins in immune responses and immunoregulation. J Allergy Clin Immunol 2015;135:598-608. [PubMed: 25592986]

7. Zenarruzabeitia O, Vitallé J, Eguizabal C, Simhadri VR, Borrego F. The biology and disease relevance of cd300a, an inhibitory receptor for phosphatidylserine and phosphatidylethanolamine. $\mathrm{J}$ Immunol 2015;194:5053-60. [PubMed: 25980030]

8. Kiwamoto T, Kawasaki N, Paulson JC, Bochner BS. Siglec-8 as a drugable target to treat eosinophil and mast cell-associated conditions. Pharmacol Ther 2012;135:327-36. [PubMed: 22749793]

9. Yokoi H, Choi OH, Hubbard W, Lee H-S, Canning BJ, Lee HH, et al. Inhibition of FcepsilonRIdependent mediator release and calcium flux from human mast cells by sialic acid-binding immunoglobulin-like lectin 8 engagement. J Allergy Clin Immunol 2008;121:499-505.e1. [PubMed: 18036650]

10. Kikly KK, Bochner BS, Freeman SD, Tan KB, Gallagher KT, D'alessio KJ, et al. Identification of SAF-2, a novel siglec expressed on eosinophils, mast cells, and basophils. J Allergy Clin Immunol 2000;105:1093-100. [PubMed: 10856141]

11. Nutku E, Aizawa H, Hudson SA, Bochner BS. Ligation of Siglec-8: a selective mechanism for induction of human eosinophil apoptosis. Blood 2003;101:5014-20. [PubMed: 12609831]

12. Nutku E, Hudson SA, Bochner BS. Mechanism of Siglec-8-induced human eosinophil apoptosis: role of caspases and mitochondrial injury. Biochem Biophys Res Commun 2005;336:918-24. [PubMed: 16157303]

13. Munitz A, Bachelet I, Eliashar R, Moretta A, Moretta L, Levi-Schaffer F. The inhibitory receptor IRp60 (CD300a) suppresses the effects of IL-5, GM-CSF, and eotaxin on human peripheral blood eosinophils. Blood 2006;107:1996-2003. [PubMed: 16254138]

14. Bachelet I, Munitz A, Moretta A, Moretta L, Levi-Schaffer F. The inhibitory receptor IRp60 (CD300a) is expressed and functional on human mast cells. J Immunol 2005;175:7989-95. [PubMed: 16339535]

15. Nicoll G, Ni J, Liu D, Klenerman P, Munday J, Dubock S, et al. Identification and characterization of a novel siglec, siglec-7, expressed by human natural killer cells and monocytes. J Biol Chem 1999;274:34089-95. [PubMed: 10567377]

16. Falco M, Biassoni R, Bottino C, Vitale M, Sivori S, Augugliaro R, et al. Identification and molecular cloning of p75/AIRM1, a novel member of the sialoadhesin family that functions as an inhibitory receptor in human natural killer cells. J Exp Med 1999;190:793-802. [PubMed: 10499918]

17. Mizrahi S, Gibbs BF, Karra L, Ben-Zimra M, Levi-Schaffer F. Siglec-7 is an inhibitory receptor on human mast cells and basophils. J Allergy Clin Immunol 2014;134:230-3. [PubMed: 24810846]

18. Yamaji T, Mitsuki M, Teranishi T, Hashimoto Y. Characterization of inhibitory signaling motifs of the natural killer cell receptor Siglec-7: attenuated recruitment of phosphatases by the receptor is attributed to two amino acids in the motifs. Glycobiology 2005;15:667-76. [PubMed: 15703304]

19. Jia Y, Yu H, Fernandes SM, Wei Y, Gonzalez-Gil A, Motari MG, et al. Expression of ligands for Siglec-8 and Siglec-9 in human airways and airway cells. J Allergy Clin Immunol 2015;135:799810.e7. [PubMed: 25747723]

20. Yamaji T, Teranishi T, Alphey MS, Crocker PR, Hashimoto Y. A small region of the natural killer cell receptor, Siglec-7, is responsible for its preferred binding to alpha 2,8-disialyl and branched alpha 2,6-sialyl residues. A comparison with Siglec-9. J Biol Chem 2002;277:6324-32. [PubMed: 11741958]

21. Miyazaki K, Sakuma K, Kawamura YI, Izawa M, Ohmori K, Mitsuki M, et al. Colonic epithelial cells express specific ligands for mucosal macrophage immunosuppressive receptors siglec-7 and -9. J Immunol 2012;188:4690-700. [PubMed: 22467657] 
22. Jandus C, Boligan KF, Chijioke O, Liu H, Dahlhaus M, Démoulins T, et al. Interactions between Siglec-7/9 receptors and ligands influence NK cell-dependent tumor immunosurveillance. J Clin Invest 2014;124:1810-20. [PubMed: 24569453]

23. Prescher H, Frank M, Gütgemann S, Kuhfeldt E, Schweizer A, Nitschke L, et al. Design, Synthesis, and Biological Evaluation of Small, High-Affinity Siglec-7 Ligands: Toward Novel Inhibitors of Cancer Immune Evasion. J Med Chem 2017;60:941-56. [PubMed: 28103033]

24. Legrand F, Tomasevic N, Simakova O, Lee C-CR, Wang Z, Raffeld M, et al. The eosinophil surface receptor epidermal growth factor-like module containing mucin-like hormone receptor 1 (EMR1): a novel therapeutic target for eosinophilic disorders. J Allergy Clin Immunol 2014;133:1439-47, 1447.e1. [PubMed: 24530099]

25. Adamko DJ, Wu Y, Ajamian F, Ilarraza R, Moqbel R, Gleich GJ. The effect of cationic charge on release of eosinophil mediators. J Allergy Clin Immunol 2008;122:383-90, 390.e1. [PubMed: 18455220]

26. Munitz A, Levi-Schaffer F. Inhibitory receptors on eosinophils: a direct hit to a possible Achilles heel? J Allergy Clin Immunol 2007;119:1382-7. [PubMed: 17337299]

27. Varchetta S, Brunetta E, Roberto A, Mikulak J, Hudspeth KL, Mondelli MU, et al. Engagement of Siglec-7 receptor induces a pro-inflammatory response selectively in monocytes. PLoS ONE 2012; 7:e45821. [PubMed: 23029261]

28. Rosenstock P, Horstkorte R, Gnanapragassam VS, Harth J, Kielstein H. Siglec-7 expression is reduced on a natural killer (NK) cell subset of obese humans. Immunol Res 2017;65:1017-24. [PubMed: 28786023]

29. Dharmadhikari G, Stolz K, Hauke M, Morgan NG, Varki A, de Koning E, et al. Siglec-7 restores $\beta$ cell function and survival and reduces inflammation in pancreatic islets from patients with diabetes. Sci Rep 2017;7:45319. [PubMed: 28378743]

30. Varchetta S, Mele D, Lombardi A, Oliviero B, Mantovani S, Tinelli C, et al. Lack of Siglec-7 expression identifies a dysfunctional natural killer cell subset associated with liver inflammation and fibrosis in chronic HCV infection. Gut 2016;65:1998-2006. [PubMed: 26674359]

31. Brunetta E, Fogli M, Varchetta S, Bozzo L, Hudspeth KL, Marcenaro E, et al. The decreased expression of Siglec-7 represents an early marker of dysfunctional natural killer-cell subsets associated with high levels of HIV-1 viremia. Blood 2009;114:3822-30. [PubMed: 19710502]

32. Landolina N, Gangwar RS, Levi-Schaffer F. Mast cells' integrated actions with eosinophils and fibroblasts in allergic inflammation: implications for therapy. Adv Immunol 2015;125:41-85. [PubMed: 25591464]

33. Carroll DJ, O’Sullivan JA, Nix DB, Cao Y, Tiemeyer M, Bochner BS. Sialic acid-binding immunoglobulin-like lectin 8 (Siglec-8) is an activating receptor mediating $\beta 2$-integrin-dependent function in human eosinophils. J Allergy Clin Immunol 2017;8;141:2196-2207

34. Zhang JQ, Biedermann B, Nitschke L, Crocker PR. The murine inhibitory receptor mSiglec-E is expressed broadly on cells of the innate immune system whereas mSiglec-F is restricted to eosinophils. Eur J Immunol 2004;34:1175-84. [PubMed: 15048729] 


\section{Key Messages}

- $\quad$ Siglec-7 is expressed at comparable levels on blood eosinophils from normal and eosinophilic subjects and expression correlates with AEC or soluble Siglec-7 levels in serum.

- $\quad$ Siglec-7 inhibits eosinophil activation but does not affect survival. 
A

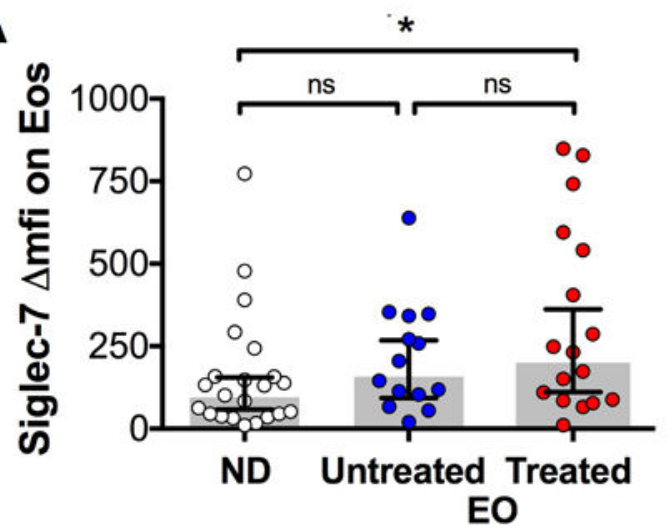

C

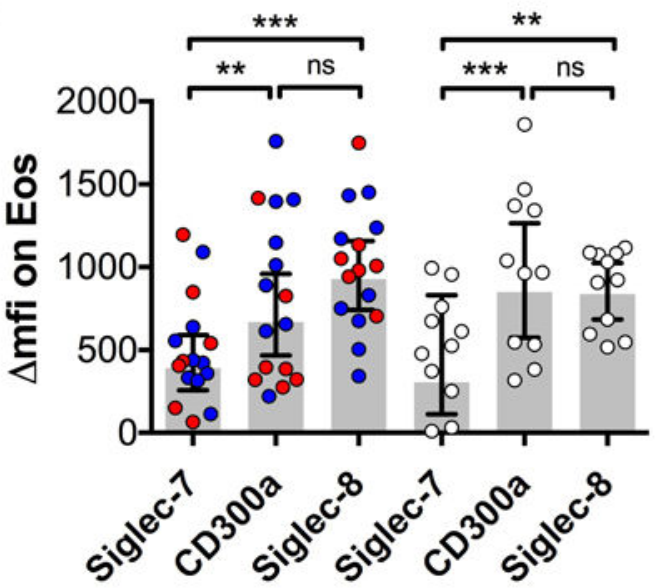

$\mathbf{E}$

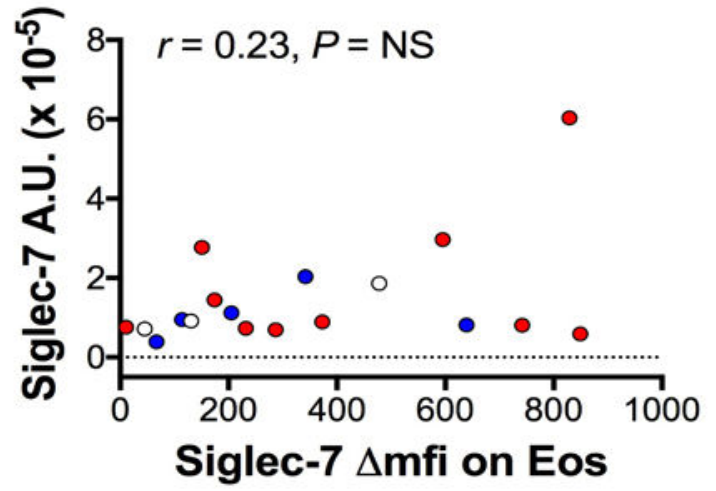

B

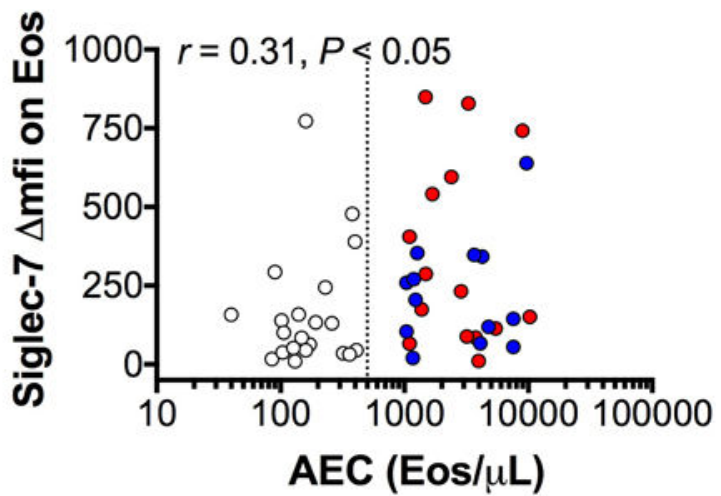

Figure 1. Siglec-7 expression on peripheral blood eosinophils.

$A$, Siglec-7 expression (expressed as $\Delta \mathrm{MFI}$ ) on eosinophils in normal donors (ND) and eosinophilic subjects (EO), untreated and treated. $B$, Correlation between Siglec-7 expression and AEC. $C$, Comparison with eosinophil expression of Siglec-8 and CD300a. $D-F$, Correlation between Siglec-7 and Siglec-8 surface expression (D), SIGLEC-7 mRNA levels and cell surface expression (E), and SIGLEC-7 and SIGLEC-8 mRNA levels (F). Symbols represent individual subjects $($ white $=$ ND, blue $=$ untreated EOS, red $=$ EOS on treatment). EOS subjects were comprised of subjects with MHES ( $n=6)$, LHES (n=6), 
Overlap HES ( $n=5)$, IHES ( $n=9)$ and other eosinophilic disorders $(n=3)$. The grey bars indicate geometric mean. Error bars represent 95\% CI. The vertical dotted line in panel B is drawn at the laboratory cutoff for eosinophilia $(500 / \mu \mathrm{L}) . * P<.05 ; * * P<.001, * * * P<.0001$. 


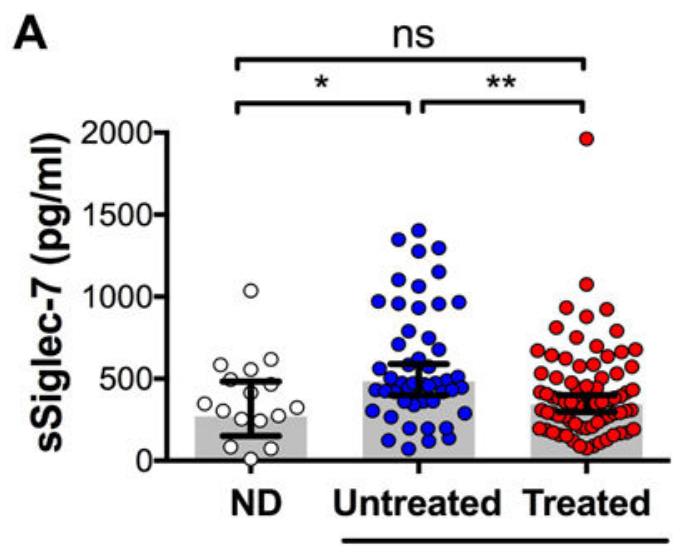

EO

C

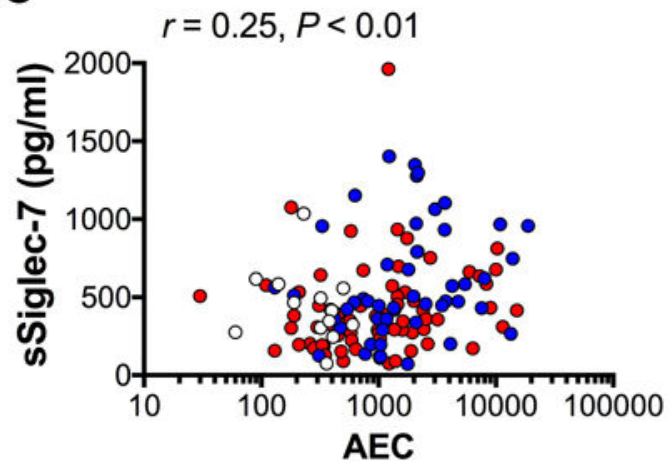

E

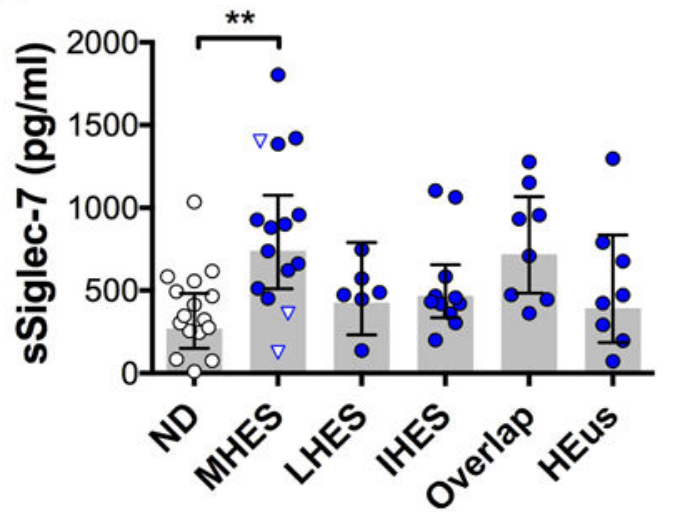

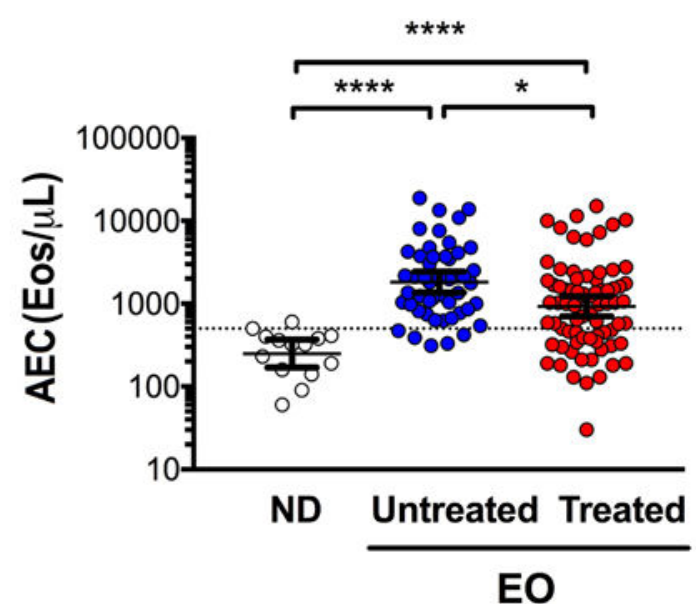

D
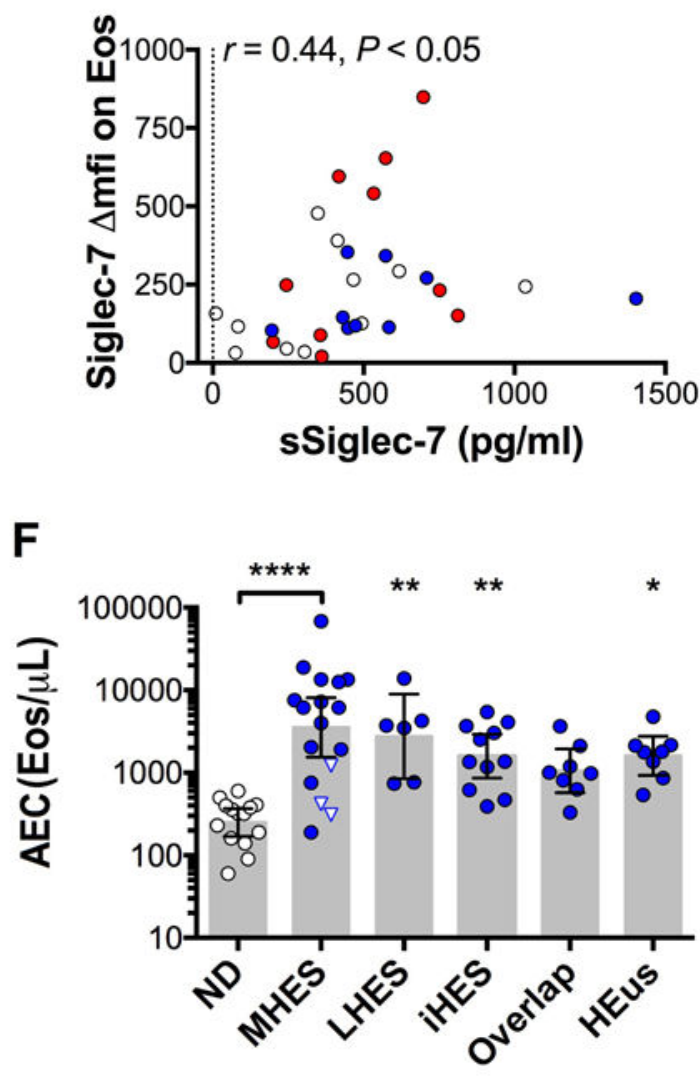

Figure 2. Serum levels of soluble Siglec-7.

$A$ and $B$, sSiglec-7 levels in serum (left) and AEC (right) from ND and EO subjects. $C$ and $D$, Comparison of sSiglec-7 levels (left) and AEC (right) between EO clinical subgroups in untreated patients (blue), inverted triangles indicate FIP1L1-PDGFRA ${ }^{\text {neg }}$ MHES. ND are shown as a control. E, Correlation of sSiglec-7 levels with AEC. F, Correlation with surface expression of Siglec-7 quantified on blood eosinophils by flow cytometry. Symbols represent individual subjects $($ white $=\mathrm{ND}$, blue $=$ untreated $\mathrm{EO}$, red $=\mathrm{EO}$ on treatment $)$. The 
grey shading indicates GM. Error bars represent $95 \%$ CI. $* P<.05 ; * * P<.001 ; * * * P<$. $0001, * * * * P<.00001$ 
A

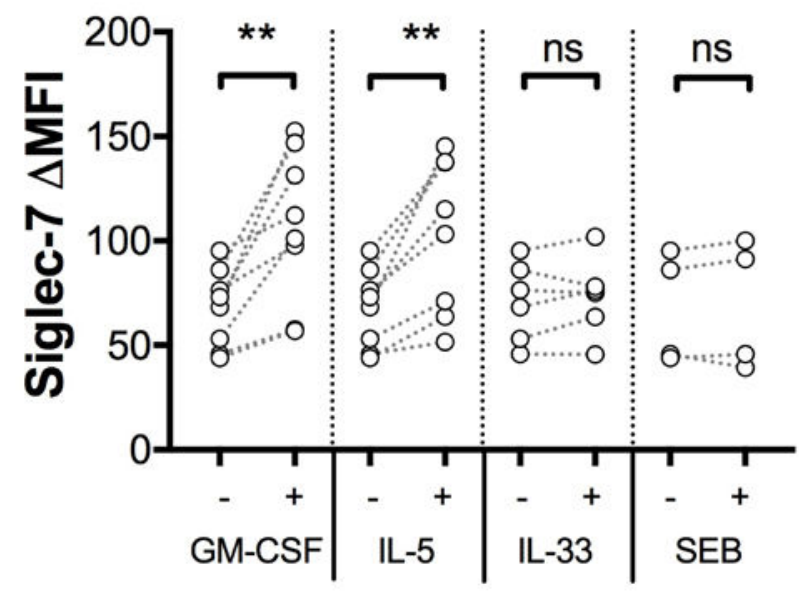

B

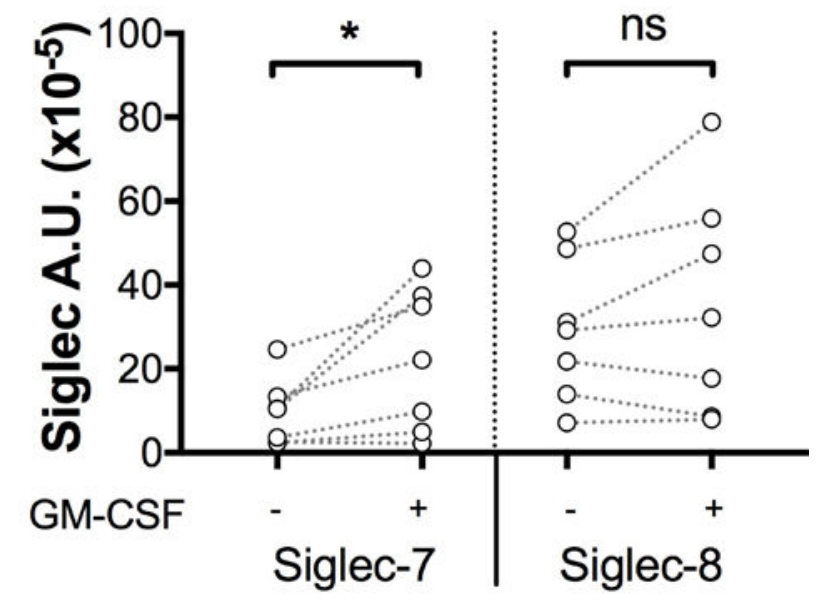

Figure 3. Siglec-7 is upregulated by stimulation with GM-CSF or IL-5.

$A$, Surface expression of Siglec-7 after overnight incubation in media (-) or media supplemented with GM-CSF, IL-5, IL-33, or SEB at 10ng/mL (+). B, SIGLEC-7 and SIGLEC-8 mRNA levels in eosinophils after overnight incubation in media (-) or media supplemented with GM-CSF at 10ng/mL $(+) .{ }^{*} P<.05(\mathrm{n}=7)$. Symbols represent individual subjects $($ white $=\mathrm{ND})$. $* * P<0.01$ 


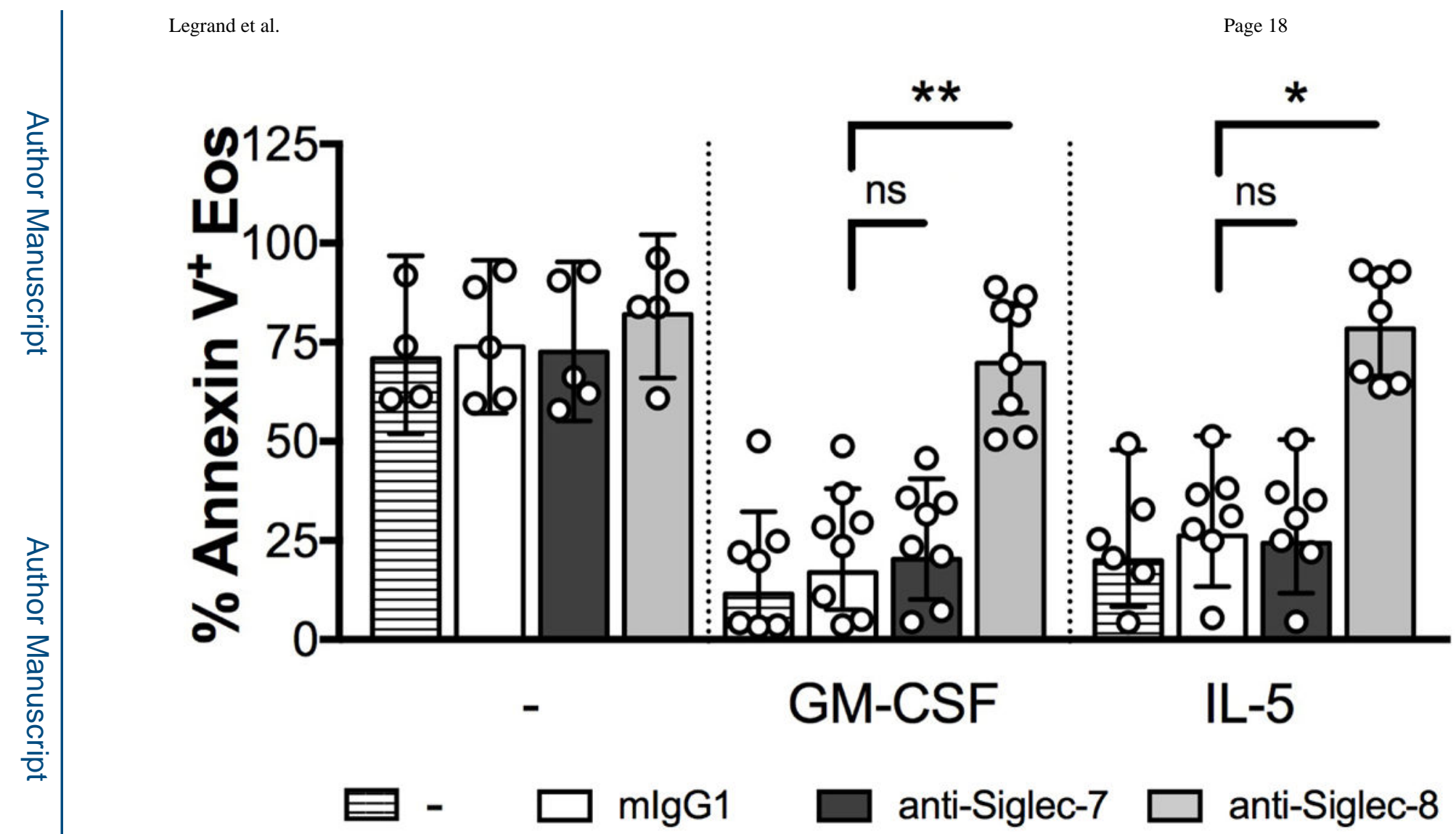

Figure 4. Siglec-7 does not induce eosinophil cell death

$A$, Eosinophil cell death after overnight incubation with GM-CSF or IL-5, eosinophils were incubated with anti-Siglec antibodies $(10 \mu \mathrm{g} / \mathrm{mL})$ for $18 \mathrm{hrs}$. Cell death was evaluated by Annexin-V/7-AAD staining and FACS analysis. Data are expressed as \% geomean with $95 \%$ $\mathrm{CI}(\mathrm{n}=8)$. Symbols represent individual subjects (white $=\mathrm{ND}) . * P<0.05, * * P<0.01$ 
A individual subjects (white $=\mathrm{ND}$ ).

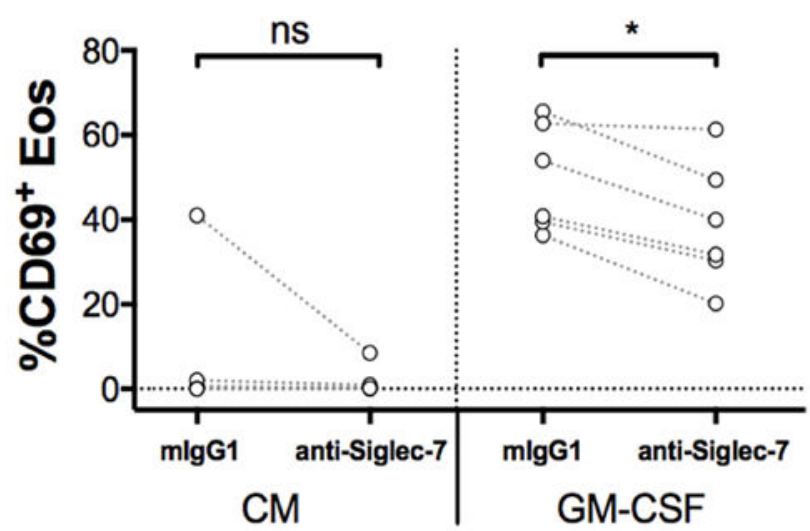

B

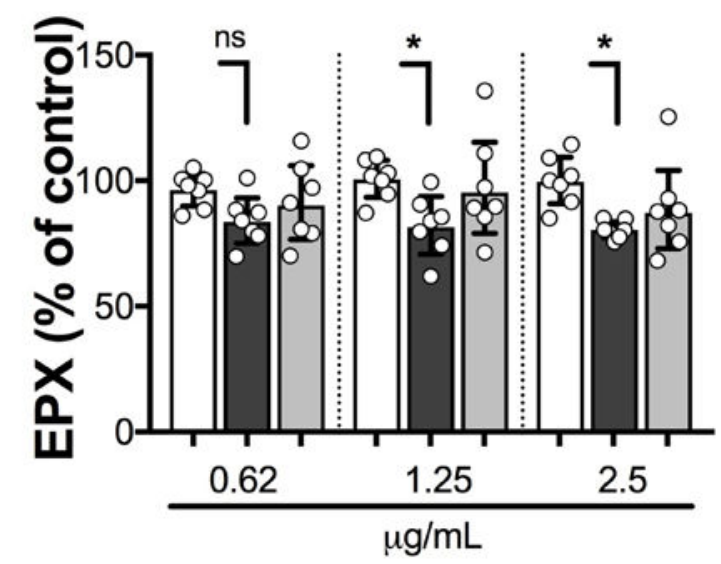

D

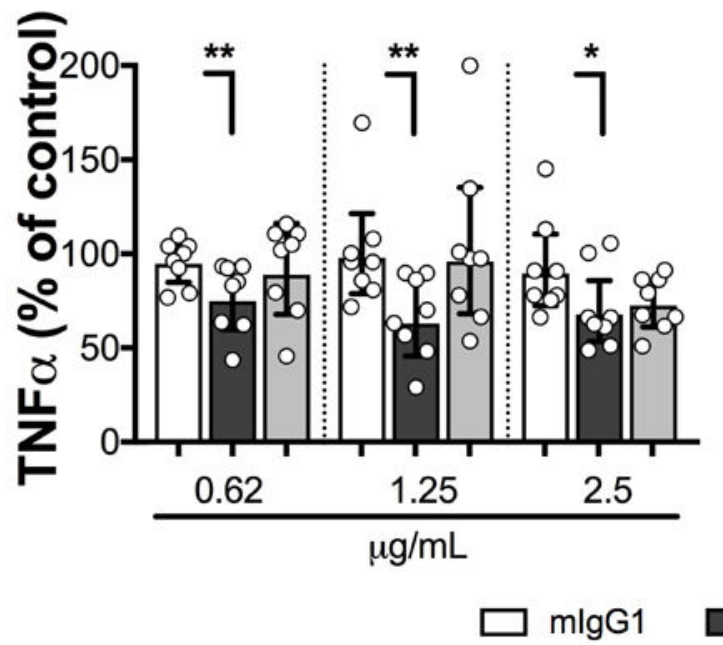

anti-Siglec-7

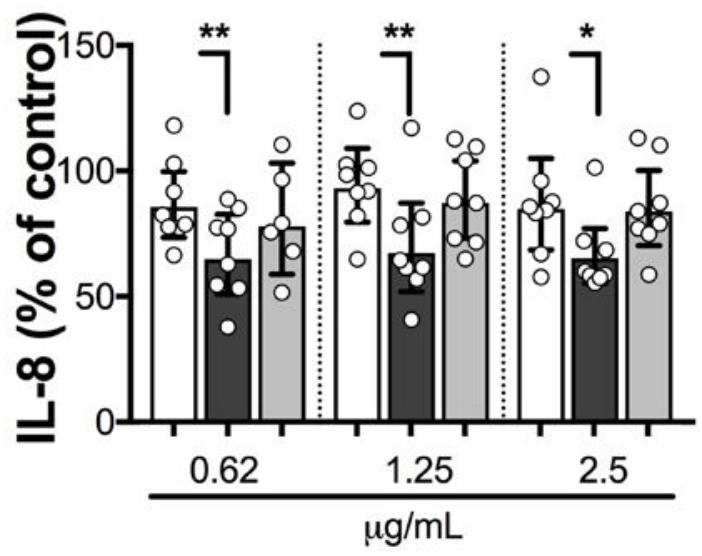

Figure 5. Crosslinking of Siglec-7 inhibits GM-CSF-induced eosinophil activation.

$A$, Eosinophil surface expression of CD69 after overnight incubation in culture medium $(\mathrm{CM})+/-\mathrm{GM}-\mathrm{CSF}(50 \mathrm{ng} / \mathrm{mL})$. The antibodies were crosslinked with $\mathrm{F}\left(\mathrm{ab}^{\prime}\right)_{2}$ goat antimouse $(10 \mu \mathrm{g} / \mathrm{mL})$. Measurement of EPX $(B)$, TNFa $(C)$ and IL-8 $(D)$ in culture supernatants following crosslinking of isotype control (white bars), anti-Siglec-7 (dark gray bars), or anti-Siglec-8 (light gray bars) at antibody concentrations ranging from 0.62-2.5 $\mu \mathrm{g} / \mathrm{mL}$ on eosinophils for 40 minute (EPX), or overnight (TNFa and IL-8) in the presence of GM-CSF ( $\mathrm{n}=7-8)$. Mediator release is expressed as the \% of the level measured in the control well containing $50 \mathrm{ng} / \mathrm{mL}$ GM-CSF alone. Data are expressed as \% GM with 95\% CI. $* P<.05 ; * * P<.001$; Wilcoxon matched-pairs signed rank test. Symbols represent 
A

C lane 1 lane 2 lane 3 lane 4 lane 5

B

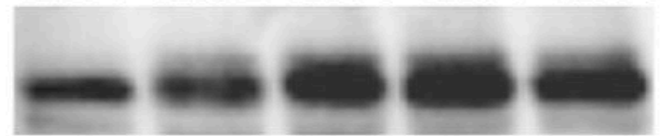

SHP-1

SHP-1

vinculin

anti-Siglec-7

anti-CD300a

CL+GM-CSF

phospho-

ERK1/2

ERK1/2

phospho-

p38

p38

GADPH

mlgG1

anti-Siglec-7

anti-CD300a

CL+GM-CSF $\begin{array}{llll}30 & 90 & 120 & 120\end{array}$

Time (seconds)
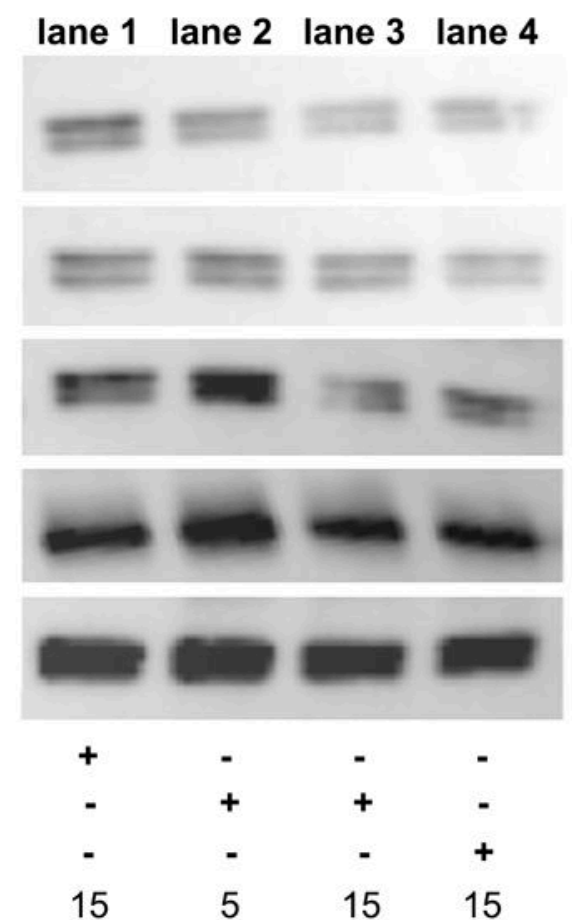

Time (minutes)
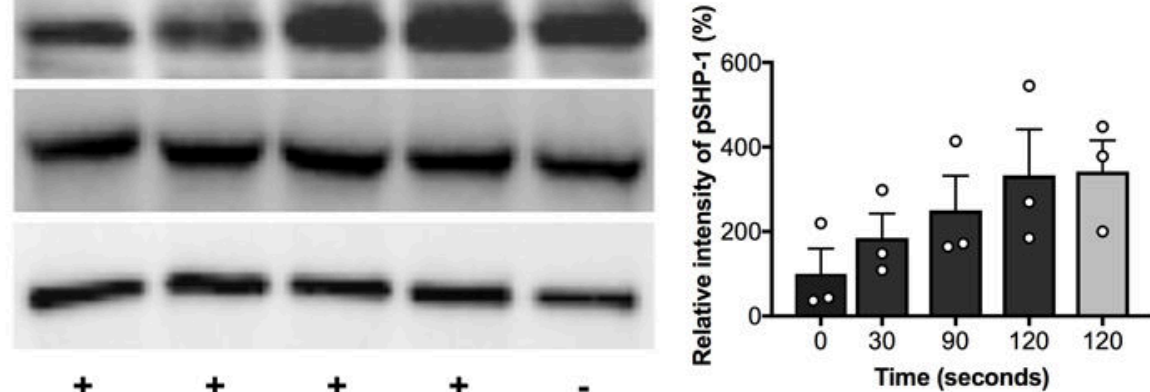

$\square$ anti-Siglec-7 $\square$ anti-CD300a

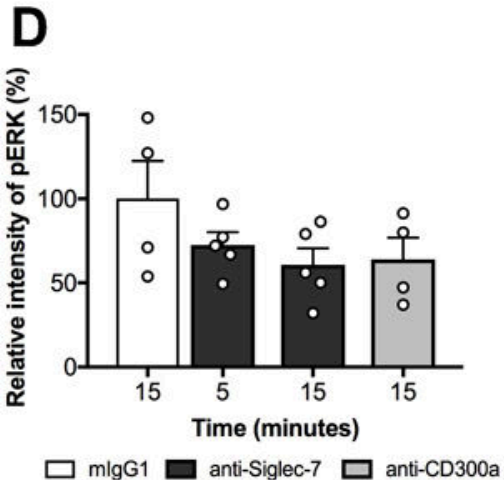

E

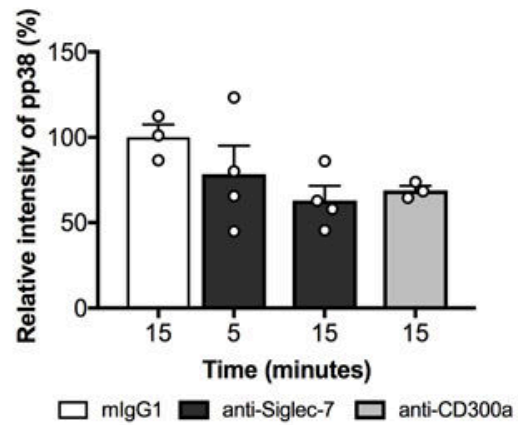

Figure 6. Activation of Siglec-7 on eosinophils induces SHP-1 phosphorylation and reduces MAPK phosphorylation in a time-dependent fashion.

Western blot detecting phosphorylation of SHP-1 ( $A$ and $B)$, ERK1/2 and p38 ( $C, D$ and $E$ ).

Eosinophils were incubated with $\mathrm{IgG} 1$, anti-Siglec-7 or CD300a antibodies $(10 \mu \mathrm{g} / \mathrm{mL})$ followed by crosslinking (CL) in the presence of GM-CSF ( $n=4)$. Phosphorylation of SHP-1 and MAPK (ERK1/2 and p38) was determined in the time interval of 0-120 sec and 5-15 min respectively. Vinculin and GAPDH were used as loading controls. Western blots shown are representative of 4 independent experiments performed on normal donors; pooled data are shown in the densitometry graphs. Relative intensity of phospho-SHP-1 $(B)$, phospho- 
ERK1/2 $(D)$ and phospho-p38 $(E)$ are shown. Symbols represent individual subjects (white $=\mathrm{ND})$. 